\title{
Work Characteristics and Upper Extremity Disorders in Female Dental Health Workers
}

\author{
Petra LindFors, Ulrica von ThIELE and Ulf LundBerG \\ Department of Psychology and Centre for Health Equity Studies, Stockholm University, Sweden
}

\begin{abstract}
Work Characteristics and Upper Extremity Disorders in Female Dental Health Workers: Petra LindFons, et al. Department of Psychology and Centre for Health Equity Studies, Stockholm University, Sweden-Many dental health workers suffer from musculoskeletal disorders in the upper extremities. In addition to ergonomic factors, psychosocial work characteristics have been linked to musculoskeletal disorders. The present cross-sectional study aimed at investigating how musculoskeletal disorders in the upper extremities (UED) and occupational position are related to work characteristics and general health problems in female dental health workers. Questionnaire data from dentists, dental hygienists and dental nurses $(N=945)$ showed that $81 \%$ reported UED. Multivariate analysis of variance showed that dentists reported the highest levels of physical load and fatigue whereas dental nurses reported the lowest levels of influence at work. Irrespective of position, those with UED considered their physical and psychosocial work environment and their own health to be significantly poorer than did those without UED. A hierarchical multiple regression showed that the physical load of dentistry was most strongly related to UED. Despite improvements to the ergonomics and physical work environment of dentistry, it is concluded that female dental health workers are still at high risk of developing UED.

(J Occup Health 2006; 48: 192-197)
\end{abstract}

Key words: Upper extremity disorders, Dentistry, Work environment, Women

Musculoskeletal disorders have repeatedly been recognized as one of the most common work-related health problems with recent research emphasizing the importance of psychosocial work characteristics in

Received Sep 13, 2005; Accepted Feb 13, 2006 Correspondence to: P. Lindfors, Department of Psychology, Stockholm University, SE-106 91 Stockholm, Sweden (e-mail:pls@psychology.su.se) addition to ergonomics and the physical work environment $^{1-3)}$. In comparison with other human-service workers, dental health workers typically report a higher incidence of work-related musculoskeletal disorders and a poorer work environment ${ }^{4-6}$.

In line with the traditional focus on ergonomic conditions and physical demands and their influences on muscular activity and the development of musculoskeletal disorders, research on dentistry has primarily considered the physical workload ${ }^{7}$. As seen in electromyographic activity, dental work is known to generate a high load, particularly on the trapezius muscle ${ }^{8,9)}$ resulting from having to work in the patient's mouth to access the teeth, which, in turn, imposes visual demands and requires finetuned and forceful movements and fixed postures for extended periods of time. Moreover, certain tasks involve exposure to vibrations and noise which increase the load even more. Furthermore, since there are no significant differences in muscle activity, posture or frequency of posture between the three most common tasks (i.e., dental examination, tooth cleaning and dental filling therapy) performed by dentists, the physical load of dental work seems to be quite stable ${ }^{5}$.

In the last decade, evidence has been accumulating pointing to relations not only between physical load and musculoskeletal disorders but also between psychosocial demands and risk of work-related musculoskeletal disorders ${ }^{1-3,10}$. Several complementary mechanisms have been suggested to explain how psychosocial stress can result in musculoskeletal tension and disorders and/or aggravate the effects of a high physical load ${ }^{3,11)}$. A central notion is that mental stress causes an increase in muscle tension similar to that caused by a physical load ${ }^{3,10,12)}$. The mental stress and cognitive demands most strongly associated with increased muscle tension and a high prevalence of musculoskeletal disorders, particularly in the upper extremities, are psychosocial factors such as time pressure, repetitive tasks, lack of control, high demands and poor social support ${ }^{1-3,10)}$. Findings regarding the psychosocial work environment of 
dentistry have been inconclusive suggesting either a poor or a satisfactory psychosocial work environment. Yet, both work environment and musculoskeletal disorders seem to vary by position and gender, and research indicates that dentists and dental hygienists have the highest demands whereas female employees have the most musculoskeletal disorders ${ }^{4,13,14)}$. However, the almost exclusive focus in recent research on dentists and dental hygienists as a group particularly exposed to work-related health-problems ${ }^{7,9,13-15)}$, has meant that the current knowledge of the work environment and health of all groups of dental health workers is limited.

The present cross-sectional study forms part of a larger project with the overall aim of investigating stress and health in dental health workers, that is dentists, dental hygienists and dental nurses, to improve their work conditions and health. In particular, the present study aimed at investigating how musculoskeletal disorders in the upper extremities (neck, shoulders, arms, elbows, wrists, hands or fingers) and occupational position are related to work characteristics, fatigue and general health disorders in female dental health workers. Hypothesizing from previous findings ${ }^{1,2,14)}$, dental health workers with upper extremity disorders were expected to report a poorer work environment in terms of physical and psychosocial work characteristics than those without such disorders.

\section{Method}

\section{Participants and data collection}

Participants were employees of the Swedish Dental Public Service in the Stockholm City County. Questionnaire data were collected by their occupational health company at 48 clinics. Participation was voluntary but encouraged by the employer. To ensure the anonymity and confidentiality, all employees were asked to complete the questionnaire individually and then return it to a representative of the occupational health company within two weeks. Thus, the employer had no access to individual questionnaires. For further details refer to "Evaluating different measures of sickness absence with respect to work characteristics"16).

All employees that were on duty and present at their workplace during the two weeks of data collection were invited to participate. In all, 1,795 of 2,025 employees, including dental health workers and other categories of personnel and excluding those on long-term absence (e.g., parental leave and long-term sick-leave), were invited to participate. The response rate was $96 \%$. The vast majority $(n=1,300)$ of the participants were dental health workers. However, for the present study, only data from female dental health workers were included $(n=945$; the response rate was about $80 \%$ ).

\section{Measures}

In addition to information on age, gender and family situation, the questionnaire also covered current position and the number of years performing current work tasks ${ }^{16)}$.

Work characteristics: Physical work characteristics were divided into physical work environment and physical load. Physical work environment was measured by five items covering computerized work, lighting, indoor climate and exposure to two types of noise. Physical load was measured by four items including monotonous movements with hands or arms, painful or strenuous work positions, heavy lifts and sedentary work. These items covered factors associated with an increased risk for work-related disorders ${ }^{17)}$. All of these items were rated along a six-point scale ranging from 'all the time' to 'never'. For both measures, additive indices were calculated with high scores indicating poor conditions.

Psychosocial work characteristics were examined using ten items rated along a six-point scale with 'yes, absolutely' and 'no, not at all' as endpoints. An initial data reduction (see Statistical analyses) was performed resulting in three components: 1) support, 2) influence at work, and 3) work-related worries. Additive indices were calculated for all three measures. For support and influence at work, low scores indicate poor conditions. For work-related worries, low scores indicate less worry.

Fatigue: Fatigue was assessed using three items covering disturbed sleep, tiredness after work and inability to stop thinking about work-related issues during subjects' free time. All items were rated along a sixpoint response format with 'low' and 'high' as endpoints. Scores on both items were added together with high scores indicating more fatigue.

Health problems: Both general health problems and musculoskeletal disorders were measured. General health problems included five items, including heartburn and stomach pain, headache, chest pains, colds and skin problems that are among the most common symptoms in the general population ${ }^{18)}$. Musculoskeletal disorders included six items covering pain in the neck or shoulder area, pain in arms or elbows, pain in wrists, hands or fingers, low back pain, pain in hips, legs or knees, and pain in feet or toes. Problems in these body areas are associated with an increased risk for work-related disorders ${ }^{17}$. For each item, respondents were asked to indicate whether or not they suffered from such health problems. Following previous research ${ }^{2}$, upper extremity disorders (UED) included problems in the neck, shoulders, arms, elbows, wrists, hands and fingers. Additive indices were calculated for general health problems and UED. The UED index was also used to categorize individuals into different groups, those with $(\mathrm{UED} \geq 1)$ or without $\mathrm{UED}(\mathrm{UED}=0)$. For both indices, high scores indicate poor health. 
Table 1. Varimax rotation of a three-factor solution for psychosocial work characteristics with loadings for each item, explained variance and internal consistency coefficients (Cronbach's alpha) for the three components

\begin{tabular}{lccc}
\hline Content of item & Support & $\begin{array}{c}\text { Component } \\
\text { Influence at work }\end{array}$ & Work-related worries \\
\hline Support from superiors & .79 & & \\
Support from co-workers & .77 & & \\
Feedback from superiors & .77 & & \\
Support from team & .62 & & \\
Overall support & .53 & .88 & .86 \\
Decide how to work & & .86 & .85 \\
Plan work & & & 15.2 \\
Time pressure & & .57 & .66 \\
Worry about change & & & \\
Insecure situation & 25.5 & .72 & \\
\hline Explained variance $(\%)$ & .77 & & \\
Cronbach's alpha & & & \\
\hline
\end{tabular}

The Kaiser-Meyer-Olkin value was .74 and above the recommended value of .6; Bartlett's Test of Sphericity reached statistical significance $(p=.0005)$.

\section{Statistical analyses}

To identify components that provide an empirically based summary of the dataset ${ }^{19}$, all items measuring psychosocial work characteristics were subjected to a principal component analysis (PCA), see Table 1 for further details.

Analyses of group differences were performed using multivariate analysis of variance (MANOVA), reporting Pillai's trace that is robust when dealing with unequal group sizes ${ }^{19}$. Significant differences were further examined by post hoc comparisons using the Bonferroni method.

A hierarchical multiple regression analysis was performed to examine the predictive value of work characteristics in relation to the number of UEDs while controlling for demographic factors and health-related measures. The first step included age, marital status ( single $=0 ;$ married/living with partner $=1$ ), position and number of years performing current work tasks. Then, fatigue and general health problems were entered into the model, and, finally, physical and psychosocial work characteristics were included. Due to missing data the degrees of freedom vary somewhat between analyses.

\section{Results}

\section{Sample characteristics}

The average age of the respondents was $45.4 \mathrm{yr}$ $(\mathrm{SD}=10.4)$. About $77 \%$ of the women were married or living with a partner. About $30 \%$ had children living at home. The majority of the women, $70 \%$, were full-time employed. Of the 945 employees, $31 \%$ were dentists,
$12 \%$ were dental hygienists and $57 \%$ dental nurses. About $81 \%$ of the women had UED, ranging from $79 \%$ in the dental nurses to $84 \%$ in the dentists, with no significant difference in prevalence between occupations.

\section{Upper extremity disorders and position as related to work} and health

Descriptive statistics for all of the work and healthrelated measures are presented in Table 2 . The analysis of the effects of UED (yes/no) and occupation (dental nurse, dental hygienist or dentist) on work characteristics showed significant main effects only. The significant effect of UED $(F(5,925)=15.81, p=.0001$, Pillai's trace $=.08$, partial eta ${ }^{2}=.08$ ) showed that employees with UED had significantly higher scores on physical work environment $(F(1,929)=42.37, p=.0001)$, physical load $(F(1,929)=64.06, p=.0001)$ and work-related worries $(F(1,929)=4.24, p=.040)$, but lower scores on influence at work $(F(1,929)=16.38, p=.0001)$ and support $(F(1$, $929)=8.92, p=.003)$ than did employees without UED. The significant effect of occupation $(F(10,1852)=14.56$, $p=.0001$, Pillai's trace $=.15$, partial eta ${ }^{2}=.07$ ) showed that dentists had significantly higher scores on physical load than did nurses $(F(2,929)=41.33, p=.0001)$. Moreover, nurses had significantly lower scores on influence at work than did the other two groups $(F(2,929)=15.83$, $p=.0001$ ).

Analysis of UED (yes/no) and occupation (dental nurse, dental hygienist or dentist) on fatigue and general health problems yielded significant main effects only. The significant effect of UED $(F(2,931)=33.99, p=.0001$, 
Table 2. Descriptive statistics for all measures for dental nurses $(n=535)$, dental hygienists $(n=116)$ and dentists $(n=294)$ with respect to upper extremity disorders (UED)

\begin{tabular}{|c|c|c|c|c|c|c|}
\hline \multirow[b]{3}{*}{ Measure } & \multicolumn{6}{|c|}{ Group } \\
\hline & \multicolumn{2}{|c|}{ Dental nurses } & \multicolumn{2}{|c|}{ Dental hygienists } & \multicolumn{2}{|c|}{ Dentists } \\
\hline & $\mathrm{M}$ & $\mathrm{SD}$ & $\mathrm{M}$ & SD & M & $\mathrm{SD}$ \\
\hline \multicolumn{7}{|c|}{ Work characteristics } \\
\hline \multicolumn{7}{|c|}{ Physical work environment } \\
\hline UED & 17.9 & 4.9 & 18.3 & 4.0 & 9.1 & 4.1 \\
\hline No UED & 15.5 & 4.1 & 15.2 & 6.1 & 4.9 & 4.9 \\
\hline \multicolumn{7}{|l|}{ Physical load } \\
\hline UED & 13.6 & 3.3 & 16.8 & 3.7 & 16.9 & 3.3 \\
\hline No UED & 11.5 & 2.9 & 13.6 & 3.4 & 13.6 & 3.6 \\
\hline \multicolumn{7}{|l|}{ Support } \\
\hline UED & 23.7 & 5.0 & 23.1 & 4.6 & 23.1 & 4.5 \\
\hline No UED & 24.8 & 4.3 & 25.3 & 3.7 & 24.3 & 4.6 \\
\hline \multicolumn{7}{|c|}{ Influence at work } \\
\hline UED & 9.9 & 3.3 & 12.0 & 3.2 & 11.1 & 3.1 \\
\hline No UED & 9.9 & 3.1 & 13.9 & 3.2 & 12.1 & 3.2 \\
\hline \multicolumn{7}{|c|}{ Work-related worries } \\
\hline UED & 5.1 & 2.7 & 4.8 & 2.6 & 4.8 & 2.7 \\
\hline No UED & 4.5 & 2.5 & 4.1 & 2.5 & 4.2 & 2.7 \\
\hline \multicolumn{7}{|c|}{ Health-related measures } \\
\hline \multicolumn{7}{|l|}{ Fatigue } \\
\hline UED & 9.8 & 3.3 & 10.3 & 3.2 & 11.7 & 3.1 \\
\hline No UED & 8.0 & 3.0 & 7.9 & 3.2 & 9.8 & 2.9 \\
\hline \multicolumn{7}{|c|}{ General health problems } \\
\hline UED & 9.3 & 1.8 & 9.5 & 1.6 & 9.6 & 1.8 \\
\hline No UED & 8.1 & 1.4 & 7.8 & 1.2 & 8.3 & 1.5 \\
\hline \multicolumn{7}{|c|}{ Musculoskeletal disorders } \\
\hline UED & 9.1 & 1.4 & 9.5 & 1.5 & 8.9 & 1.4 \\
\hline No UED & 6.4 & 0.7 & 6.3 & 0.5 & 6.4 & 0.7 \\
\hline
\end{tabular}

Table 3. Significant predictors of upper extremity disorders

\begin{tabular}{lcccc}
\hline Step & Predictor & Beta & $t$ & $p$ \\
\hline Step 1 & Age & .13 & 4.16 & .0001 \\
Step 2 & General health problems & .22 & 5.94 & .0001 \\
Step 3 & Physical load & .24 & 6.61 & .0001 \\
& Physical work environment & .09 & 2.31 & .021 \\
\hline
\end{tabular}

Only significant predictors are included in the table. Neither marital status, position, number of years performing current work tasks (entered in Step 1), fatigue (entered in Step 2), nor psychosocial work characteristics (entered in Step 3), remained significant.

Pillai's trace $=.07$, partial eta $\left.{ }^{2}=.07\right)$ showed that women with UED reported significantly more fatigue $(F(1,932)=$ $33.69, p=.0001)$ and general health problems $(F(1,932)=$ $60.15, p=.0001)$ than did women without UED. The significant effect of occupation $(F(4,1864)=10.12$, $p=.0001$, Pillai's trace $=.04$, partial eta $\left.{ }^{2}=.02\right)$ showed that dentists had significantly higher scores on fatigue than did the other two groups of employees $(F(2,932)=19.38$, $p=.0001$ ).

\section{Predictors of upper extremity disorders}

Table 3 summarizes the results from the hierarchical regression analysis which showed that physical load, general health problems, age and physical work 
environment were significantly associated with the number of UED. More specifically, a higher physical load, more general health problems, increasing age and a poorer physical work environment were associated with UED. However, neither marital status, position, number of years performing current tasks, fatigue nor the psychosocial work characteristics made a unique contribution to the model. Between the different steps, the $\mathrm{R}^{2}$ change ranged from .07 to .10 with values for $F$ change ranging from 14.9 to $51.47(p=.0001)$. The model as a whole explained $18 \%$ of the variance (adjusted $\left.\mathrm{R}^{2}=.17\right)$ and yielded a significant $\operatorname{ANOVA}(F=19.30$, $p=.0001)$.

\section{Discussion}

In line with previous studies on dentists ${ }^{14,20)}$, the present findings showed that a large majority $(81 \%)$ of the female dental health workers reported UED. This figure is somewhat higher than that reported in a recent study on the prevalence of musculoskeletal disorders in dentists ${ }^{20)}$ and it is about $10-20 \%$ higher than in other high-risk occupations $^{22)}$. Despite working within the same organization and in clinics meeting the standards of a modern work environment, it was found that female employees with UED rated the characteristics of their work as significantly poorer and also reported significantly more fatigue and general health problems than did those without UED. The results are also in line with previous studies in which dentists with musculoskeletal disorders reported less work satisfaction, more anxiety and poorer health than did those without such disorders ${ }^{14)}$. However, the present study also shows that the physical load, including precision work in strenuous positions, and general health problems, such as headaches and stomach pains, are the factors most strongly related to UED. Thus, the psychosocial work characteristics appear to be of less significance than the physical work characteristics, which may be connected with the physical load in dentistry being so heavy that it overshadows the acute effects of the psychosocial work characteristics on UED.

Moreover, the present findings showed that dentists reported a higher physical load than did dental nurses, whereas dental nurses reported the lowest influence at work (Table 2). As suggested by previous research ${ }^{5}$, these findings most likely reflect the fact that the dentist, when working on a patient's teeth, experiences a physical load as a result of the fine-tuned movements and strenuous work positions needed to reach the teeth area. During their work, the dentist decides and plans the subsequent steps in the treatment and in doing so determines the tasks and the work pace of the assisting dental nurse who, consequently, has less influence at work. However, the present findings also showed that dentists reported the highest levels of fatigue. In line with the mechanisms suggested by previous research ${ }^{3,10)}$, this probably results from the combination of physical load and mental stress (influence at work), that is, working in strenuous positions while deciding on and planning the work and communicating this to the dental nurse. Like the dentists, the dental hygienists also have high levels of influence at work (Table 2). Yet, in contrast to the dentists, the dental hygienists usually meet with the patient on a one to one basis and this may explain why the dental hygienists show lower levels of fatigue after work. Even though the work of the dental hygienist is performed without the immediate presence of a colleague, it should be noted that there were no significant differences in support between the different categories of dental health workers.

This study included only employees from the Swedish Dental Public Service and this may make generalization to private dental clinics difficult. However, even though the public clinics are usually larger and employ more women than do private clinics, the tasks of dentistry and the resulting physical load ${ }^{5,8)}$ are similar in these two organizational settings. Moreover, the physical load was strongly associated with UED which suggests that the results are highly relevant for those employed in private dental clinics as well. However, the conclusions concerning the causal directions between workload and health are, of course, limited due to the cross-sectional design. Also, the concurrent assessment of self-ratings of work characteristics and health problems may increase the risk for shared response bias, which contributes to an overestimation of associations between workload and health-related outcomes ${ }^{21)}$. Due to the reliance on selfreports, there is always a possibility that individuals not reporting any UED have had them for such a long time that they have grown used to them and do not acknowledge the existence of them. However, this is unlikely since the percentages of individuals with and without musculoskeletal problems are comparable to those reported in other studies. In line with previous research ${ }^{2)}$, the present study used a sum scale of UED. However, additional analyses (results not shown) of musculoskeletal problems in specific body regions yielded comparable results thus showing that female dental health workers reporting UEDs seem to have pain in several body regions.

To conclude, the findings from the present study once again highlight that the occupational group of female dental health care workers, and particularly female dentists, are at risk of developing musculoskeletal disorders and are therefore likely to benefit from additional improvements of the work environment, targeted prevention and intervention aimed at reducing these risks.

Acknowledgments: Thanks to the employees at the 
Swedish Dental Public Service in Stockholm City County and to Previa. This research was supported by grants to Ulf Lundberg from the Swedish Council for Working Life and Social Research, and to Petra Lindfors from the Anna Ahlström and Ellen Terserus Foundation.

\section{References}

1) PM Bongers, CR de Winter, MAJ Kompier and VH Hildebrandt: Psychosocial factors at work and musculoskeletal disease. Scand J Work Env Health 19, 297-312 (1993)

2) PM Bongers, AM Kremer and J ter Laak: Are psychosocial factors, risk factors for symptoms and signs of the shoulder, elbow or hand/wrist? A review of the epidemiological literature. Am J Ind Med 41, 315-342 (2002)

3) U Lundberg: Psychophysiology of work: Stress, gender, endocrine response and work-related upper extremity disorders. Am J Ind Med 41, 383-392 (2002)

4) E Bejerot: Dentistry in Sweden-Healthy work or ruthless efficiency? Arbete och Hälsa [Work and Health] 14, 1-66 (1998)

5) L Finsen, H Christensen and M Bakke: Musculoskeletal disorders among dentists and variation in dental work. Appl Ergonomics 29, 119-125 (1998)

6) I Åkesson, B Johnsson, L Rylander, U Moritz and S Skerfving: Musculoskeletal disorders among female dental personnel—clinical examination and a 5-year follow-up study of symptoms. Int Arch Occup Env Health 72, 395-403 (1999)

7) BL Rundcrantz, B Johnsson and U Moritz: Pain and discomfort in the musculoskeletal system among dentists. A prospective study. Swed Dent J 15, 219228 (1991)

8) E Milerad, MO Ericson, R Nisell and Å Kilbom: An electromyographic study of dental work. Ergonomics 34, 953-962 (1991)

9) T Öberg, A Karsznia, L Sandsjö and R Kadefors: Work load, fatigue, and pause patterns in clinical dental hygiene. J Dent Hyg 69, 223-229 (1995)

10) B Melin and U Lundberg: A biopsychosocial approach to work-stress and musculoskeletal disorders. J Psychophysiol 11, 238-247 (1997)

11) GD Huang, M Feuerstein and SL Sauter: Occupational stress and work-related upper extremity disorders:
Concepts and models. Am J Ind Med 41, 298-314 (2002)

12) G Sjøgaard, U Lundberg and R Kadefors: The role of muscle activity and mental load in the development of pain and degenerative processes on the muscle cellular level during computer work. Eur J Appl Physiol 83, 99-105 (2000)

13) H Murtomaa, E Haavio-Mannila and I Kandolin: Burnout and its causes in Finnish dentists. Comm Dent Oral Epidemiol 18, 208-212 (1990)

14) BL Rundcrantz, B Johnsson, U Roxendal and U Moritz: Occupational cervico-branchial disorders among dentists. Psychosocial work environment, personal harmony and life-satisfaction. Scand J Soc Med 19, 174-180 (1991)

15) V Ylipää, BB Arnetz and H Preber: Predictors of good general health, well-being and musculoskeletal disorders in Swedish dental hygienists. Acta Odont Scand 57, 277-282 (1999)

16) U von Thiele, P Lindfors and U Lundberg: Evaluating different measures of sickness absence with respect to work characteristics. Scand J Publ Health (in press)

17) Swedish Work Environment Authority. Work-related disorders. Swedish Official Statistics, Statistical Report No. AM43 SM04011. Available from: http:// www.av.se/statistik/dok/0000286.pdf (Accesed January $10,2005)$.

18) G Tibblin, C Bengtsson, B Furunes and L Lapidus: Symptoms by age and sex: The population studies of men and women in Gothenburg, Sweden. Scand J Prim Health Care 8, 9-17 (1990)

19) Tabachnick BG and Fidell LS: Using multivariate statistics (4th ed.). Boston, MA: Allyn and Bacon, 2000.

20) EC Alexopoulos, I-C Stathi and F Charizani: Prevelance of musculoskeletal disorders in dentists. BMC Musculoskel Dis 5, 16-23 (2004)

21) JJ Hurrell, DL Nelson and BL Simmons: Measuring job stressors and strains: Where we have been, where we are, and where we need to go. J Occup Health Psychol 3, 368-389 (1998)

22) D Rissén, B Melin, L Sandsjö, I Dohns and U Lundberg: Surface EMG and psychophysiological stress reactions in women during repetitive work. Europ J Appl Phys 83, 215-222 (2000) 